\title{
Detecting gas-liquid two-phase flow pattern determinism from experimental signals with missing ordinal patterns
}

\author{
Meng Du, ${ }^{1, \text { a) }}$ Lei Zhang, ${ }^{1, \text { b) }}$ Xiangyang Niu, ${ }^{1, \mathrm{c})}$ and Celso Grebogi ${ }^{2, \mathrm{~d})}$ \\ 1) the school of Electrical Engineering and Automation, Tianjin University of science and technology, Tianjin 300222, \\ China \\ ${ }^{2)}$ the Institute for Complex Systems and Mathematical Biology, King's College, University of Aberdeen, Aberdeen AB24 $3 U E$, \\ $U K$
}

To address the issue of whether there exists determinism in a two-phase flow system, we first conduct a gas-liquid two-phase flow experiment to collect the flow pattern fluctuation signals. Then we investigate the determinism in the dynamics of different gas-liquid flow patterns by calculating the number of missing ordinal patterns associated with the partitioning of the phase space. In addition, we use the recently proposed stretched exponential model to reveal the flow pattern transition behavior. With the joint distribution of two fitted parameters, which are the decay rate of the missing ordinal patterns and the stretching exponent, we systematically analyze the flow pattern evolutional dynamics associated with the flow deterministic characteristics. This research provides a new understanding of the two-phase flow pattern evolutional dynamics and broader applications in more complex fluid system is suggested.

\begin{abstract}
The two-phase flow, such gas and liquid, is a frequently encountered dynamical system in many fields, such as nuclear, petrochemical, biochemical, hydraulic, and others. A fundamental problem of the two-phase flow system is the presence of both determinism and stochasticity in its dynamics. Understanding the two-phase flow characteristics and its deterministic evolutionary dynamics is of importance and of continuing interest for the flow control system optimization. For that, we need to characterize the system determinism, what we accomplish by partitioning the phase space, assigning symbols to each partition, and then counting the number of missing ordinal patterns (NMP) during the system's evolution. This is a sensitive and a reliable index to characterize the system's determinism. We design an experimental gas-flow facility and carry out the experiments by exploring parameter space of possible flow conditions. The collected fluctuation data are then employed to assess determinism using our NMP method. We further investigate the NMP scaling behavior of the flow fluctuations to demonstrate the diverse gasliquid two-phase flow evolutionary dynamics. The results of this work show that the gas-liquid two-phase flow deterministic dynamics is associated with a phase morphology distribution, called the flow patterns. The methods used in this work are expected to apply in more complex fluid systems such as multi-phase flow and microfluidics.
\end{abstract}

\section{INTRODUCTION}

Gas-liquid two-phase flow widely exists in industrial processes such as crude oil production, nuclear-power generation, fluidization, and so on. How to clearly characterize the com-

\footnotetext{
a) Electronic mail: mdu@tust.edu.cn

b)Electronic mail: z186481900zl@163.com

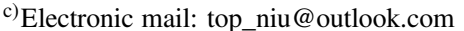

d)Electronic mail: grebogi@abdn.ac.uk
}

plex flow pattern structure and to reveal its evolutional dynamics are fundamental problems that are frequently encountered in two-phase flow industry applications such as pipeline pressure drop prediction, flow rate measurement and process parameter optimization.

Experimental observation is the most frequently used method to reveal the gas-liquid two-phase flow pattern dynamics. Various observation tools, such as high-speed camera, ${ }^{1}$ tomograph,${ }^{2}$ particle image velocimetry, ${ }^{3,4}$ contactless sensor, ${ }^{5}$ and infrared transceiver, ${ }^{6}$ are used to discover the flow pattern structure and dynamics. The experimental observations can provide intuitive flow pattern information and it is rather straightforward to establish the flow pattern transition criteria based on the observed phenomena. However, the experimentally observed flow pattern information is typically not enough to clearly demonstrate the underlying flow pattern mechanism. It means that we still need more effective methods to characterize the phenomena of phase interaction and flow pattern transition. To reveal the underlying flow pattern evolutional dynamics, some researchers make use of the computational fluid dynamics (CFD) method to study the gas-liquid two-phase flow dynamics. Various CFD models have been developed to characterize the gasliquid two-phase flow in vertical pipes, ${ }^{7}$ horizontal pipes, ${ }^{8}$ inclined pipes, ${ }^{9}$ porous media, ${ }^{10}$ and micro-channels. ${ }^{11}$ Noteworthy comparisons between the CFD simulated dynamics and the experimental results are reported to give a better understanding of the gas-liquid two-phase flow system. ${ }^{12,13}$ Another tool that can effectively characterize the flow pattern evolutional dynamics is the experimental collected fluid fluctuations. ${ }^{14}$ Generally, the two-phase flow fluctuations reflect the measured sequential pressure differentials ${ }^{15,16}$ or the mixture conductivity. ${ }^{17}$ Various time series analysis methods, including spectrum analysis, ${ }^{18,19}$ joint time-frequency distribution, ${ }^{20}$ wavelet analysis, ${ }^{21}$ Hilbert-Huang transform, ${ }^{22}$ nonlinear analysis, ${ }^{23,24}$ recurrence plot analysis, ${ }^{25}$ complex network analysis, ${ }^{26,27}$ and machine learning, ${ }^{28-31}$ have been applied to reveal different aspects of flow pattern dynamics. Several reflective quantitative indexes such as energy, ${ }^{32}$ entropy, ${ }^{33}$ and time irreversibility ${ }^{34}$ have been applied to char- 
acterize the flow patterns.

A fundamental problem of the two-phase flow system is when there exists either determinism or the flow pattern evolution is an entirely stochastic process. From the collected fluctuation signals point of view, some flow pattern fluctuations, such as that of bubble flow, show random features and the deterministic dynamics may be masked by the noisy-like signals. Hence, it is necessary to develop a reliable method to detect the flow pattern determinism from collected noisy fluctuation signals. Recently, using permutation parameters to detect the system nonlinearity and determinism via ordinal patterns has attracted much attention. ${ }^{35-37}$ For example, Kulp and Zunino, ${ }^{38}$ propose a permutation spectrum test method which uses the standard deviation of the permutation spectra to characterize the system chaotic and stochastic dynamics. After the phase space is symbolized with the ordinal patterns, the influence of noise can be reduced and useful deterministic information is effectively extracted. The ordinal pattern related methods have now been proved to be powerful tools in the field of financial, ${ }^{39}$ biomedical, ${ }^{40,41}$ interdependencies detection, ${ }^{42,43}$ hydrology, ${ }^{44}$ and so forth. Moreover, some researchers combine the ordinal pattern analysis with other dynamic detection techniques, such as recurrence quantification analysis, ${ }^{45}$ complex network analysis, ${ }^{46}$ and multiscale analysis, ${ }^{47}$ to reveal more sophisticated system dynamics. In this regard, establishing the bridge of flow patterns and the experimental fluctuation ordinal patterns is an appealing and a rewarding approach to reveal the complex flow pattern evolutional dynamics. In addition, the ordinal pattern related methods also provide reliable and applicable ways to evaluate the flow pattern determinism.

The remainder of this paper is organized as follows. In the next Section, we introduce the concept of missing ordinal patterns. A recently proposed stretched exponential model ${ }^{48}$ is also introduced. This model is further used to characterize the flow pattern determinism evolutional dynamics. In Section III, we describe our gas-liquid two-phase flow experiment in a vertical upward pipe. The procedure to collect the flow pattern conductance fluctuation signals is also described. Section IV shows the results by using the methods described in the previous Section. The conclusions are in Section V.

\section{METHOD}

The concept of ordinal patterns of a trajectory ${ }^{49}$ has been shown to be a powerful tool for detecting the dynamics of complex systems. ${ }^{50,51}$ In this method, one divides the phase space into partitions. One then assigns a unique symbol to each partition. In this way, the system is symbolized and the trajectory is given by a sequence of symbols, ${ }^{52}$ having diverse ordinal patterns. The dynamics, even masked by environmental and observation noise can be revealed in this symbolic space.

Consider a time series $x(t)$, which is collected by observing the system dynamics. We first reconstruct the phase space vector $\vec{X}(t)$ with the standard delay coordinate embedding method. ${ }^{53}$

$$
\vec{X}(t)=[x(t), x(t+\tau), \cdots x(t+(m-1) \tau)]
$$

where $m$ denotes the embedded dimension and $\tau$ refers to the embedding delay time. Each element of $\vec{X}(t)$ is assigned a number from 1 to $m$, which is related to the value of the partition. Then, we can obtain a permutation of the symbols, which can be expressed by a sequence as follows:

$$
\pi_{i}=\left[k_{1}, k_{2} \ldots k_{m}\right]
$$

where $k_{1}, k_{2} \cdots k_{m}$ denotes the given permutation and $\pi_{i}$ refers to the symbol sequence related to the $\mathrm{i}$-th vector to be reconstructed. Given an m-dimensional system, there exists $m$ ! possible permutations or ordinal patterns. Take $m=3$, for example, there exits a total of six ordinal patterns, which are shown in Fig. 1.

FIG. 1. Six ordinal patterns when the reconstruction dimension is $m=3$.

For a sufficiently long time series generated from a completely stochastic system, all the ordinal patterns are observed. However, some ordinal patterns are absent in the reconstructed symbolic sequence from some nonlinear deterministic systems. Although some deterministic systems, like Anosor diffeomorphisms, ${ }^{54}$ all the symbols are present. The absent patterns are defined as forbidden patterns. The Number of Forbidden Patterns (NFP) has been shown to be a powerful indicator to reveal the determinism of the generated time series. ${ }^{55}$ To get a reliable estimation of the NFP, the length of time series $x(t)$ needs to satisfy $N \gg m$ ! and the embedded dimension $m$ is to be chosen in the range of 3 to 7 . One difficulty when using the NFP is that the time series needs to be long 
enough to obtain all the possible ordinal patterns. However, it is not certain that, when involving shorter time series, whether the presence of missing ordinal patterns is caused by the system determinism or caused by the small sample effect. Fortunately, recent research has shown that the incomplete number of missing ordinal patterns (NMP) in short time series can still be used as an effective indicator to identify the system nonlinearity and determinism. ${ }^{56}$

The deterministic component of a complex system can be obtained by the scaling behavior of NMP with length of the time series. The NMP decreases when increasing the observed data length. ${ }^{57}$ The exponential function is frequently used to fit the relationship between the NMP and the time series length. Recently, a stretched exponential model ${ }^{48}$ has been shown to be more suitable for modeling the correlated stochastic data. This descriptive model can be expressed as,

$$
N M P=A \cdot \exp ^{\left(-B \omega^{\gamma}\right)}
$$

where $A$ is a constant and $\omega$ refers to the length of the time series. $B$ and $\gamma$ are the two stretched exponential parameters, reflecting the determinism of the system. The decay rate $B$ indicates how fast the NMP decreases. A faster decay rate of the NMP means that the system tends to be more stochastic, leading to a smaller value of $B$. The stretching exponent $\gamma$ increases when the system evolves from a relatively deterministic state to a more stochastic one. Note that when $\gamma=1$, (3) reduces to the exponential decay rate, meaning that the system is completely uncorrelated. In Section IV, the use of the number of missing ordinal patterns to characterize flow pattern nonlinearity and determinism is demonstrated. With the joint analysis of the decay rate $B$ and the stretching exponent $\gamma$, the evolutional dynamics of gas-liquid flow pattern in a vertical pipe is investigated.

\section{EXPERIMENTS AND DATA ACQUISITION}

\section{A. Experimental setup and procedure}

We conduct a gas-liquid two-phase flow experiment in a flow loop to collect fluctuation data. These fluctuations reflect the flow pattern dynamics under different flow conditions. As shown in Fig. 2, the flow loop consists of a water tank, an air pump, a peristaltic metering pump, and a section of $50 \mathrm{~mm}$ inner diameter acrylic plastic testing pipe.

In the experiment, we first introduce the water phase into the vertical testing pipe, and then the gas phase is added to generate the desired gas-liquid flow patterns. When the flow pattern is fully developed, conductance fluctuation signals of the mixed fluid are measured with a four-ring conductance sensor ${ }^{58}$ which is fixed on the vertical testing pipe. As shown in Fig. 2, the conductance sensor consists of four stainless steel conductance rings, which are axially separated and fixed on the inside wall of the testing pipe. E1 and E2 are the excitation electrodes that are connected to a $100 \mathrm{kHz}$ sinusoidal signal. M1 and M2 are the measuring electrodes that the conductance fluctuating signals measured from M1 and M2 are
FIG. 2. Schematic of gas-water flow loop facility

mainly correlated with the fluid fluctuations and dynamics. The axial height of the excitation and measuring electrodes are $6 \mathrm{~mm}$ and $4 \mathrm{~mm}$, respectively. The distance between M1 and $\mathrm{M} 2$ is $82 \mathrm{~mm}$, and the distance between E1 and E2 is $240 \mathrm{~mm}$. Since the excitation voltage on the electrodes E1 and E2 is constant sinusoidal signal, the measured differential voltage on M1 and M2 reflects the two-phase flow volume fraction fluctuations. The conductance fluctuation signals of the mixed gas-liquid flow are collected by a data acquisition system with the sampling rate set at $2000 \mathrm{~Hz}$. In addition, we collect a series of flow snapshots with a high-speed camera to observe the flow patterns. The water flow rate is controlled with the metering pump, and the gas flow rate is measured with a gas flow meter and controlled with a valve. The water flow velocity is set in the range from $0.0019 \mathrm{~m} / \mathrm{s}$ to $0.1953 \mathrm{~m} / \mathrm{s}$ and the gas flow velocity is set between $0.0018 \mathrm{~m} / \mathrm{s}$ and $0.0207 \mathrm{~m} / \mathrm{s}$. A total of 284 flow conditions are tested in this experiment.

\section{B. Observed flow patterns and the fluctuation signals}

With the aid of high-speed camera, we observe three typical gas-liquid flow patterns in the vertical pipe: slug flow, non-uniform bubble flow, and uniform bubble flow. ${ }^{59}$ Typical flow pattern snapshot series are shown in Fig. 3. Gas-liquid slug flow is a kind of flow pattern that show some pseudoperiodic behavior. When the water velocity is very low, as shown in Fig. 3(a), large gas slugs (Taylor bubbles) with the diameter almost equal to the pipe diameter intermittently pass through the vertical testing pipe. We also find that each Taylor bubble is followed by a wake, which captures a cluster of 
small gas bubbles. When increasing the water velocity, the flow pattern evolves from slug to non-uniform bubble flow. The snapshot series of non-uniform bubble flow are shown in Fig. 3(b). Under this situation, the gas phase is coalesced into large bubbles, which are non-uniformly distributed. Bubble clusters and large spherically capped bubbles frequently appear in the vertical pipe. With even higher water velocity, the turbulence energy of the mixed fluid is high enough to break up the gas phase into smaller and uniformly distributed bubbles. Then, the flow pattern changes to uniform bubble flow. As shown in Fig. 3(c), the global structure of this flow pattern is stable while the dynamics of the dispersed gas bubbles show stochastic characteristics.
FIG. 3. Snapshot series of different flow patterns. (a) Slug flow, (b) Non-uniform bubble flow, and (c) Uniform bubble flow.

The typical flow pattern fluctuation signals are shown in Fig. 4. As we can see in Figs. 4(a)-(c), the fluctuations of the slug flow exhibit apparent pseudo-periodic features, reflecting the intermittent motion of the gas slugs. The amplitude of the fluctuation peaks reflects the size of the gas slugs. Figs. 4(d)-(f) show the conductance signals of non-uniform bubble flow. The amplitude of the flow pattern fluctuations is smaller than that of the slug flow, indicating that the gas phase evolves to smaller structures. We also notice that there exist obvious intermittent peaks in the fluctuation signals of non-uniform bubble flow, reflecting the frequent appearance of spherically capped bubbles or bubble clusters. The conductance fluctuation signals of uniform bubble flow is shown in Figs. 4(g)(i). As we can see, these signals are noise like which reflect the stochastic features of the discrete gas bubbles. Also, the amplitude of the uniform bubble flow fluctuations are smaller than that of the other two flow patterns, which means that the structure of the uniform bubble flow becomes more stable due to smaller gas bubble size at higher water velocity.
FIG. 4. The typical conductance fluctuation signals from different flow patterns. (a)-(c)Slug flow, (d)-(f)Non-uniform bubble flow, and (g)-(i)Uniform bubble flow

\section{RESULTS AND DISCUSSION}

\section{A. Missing ordinal patterns of gas-liquid two-phase flow signals}

The number of missing patterns (NMP) reflects the presence of determinism in the dynamics of a complex system. We in this work, use the NMP as an index to reveal the determinism of different gas-liquid two-phase flow patterns. When calculating the NMP of flow pattern fluctuations, the duration of the time series is set at 100 seconds, that is, 200000 points for each fluctuation signal. It is suggested that the value of $m$ is chosen in the range of 3 to $7 .{ }^{56}$ However, the $P_{N M P}$ is becoming insensitive to the flow pattern fluctuations when the value of $m$ is too small. Take $m=3$ for example, there only exists 6 ordinal patterns that is hard to distinguish the flow pattern determinism dynamics with so few ordinal patterns. In this regard, we in this paper calculated the NMP when the embeded dimension is set as 5, 6, and 7. We choose a commonly used value $\tau=1$, according to previous study. ${ }^{56}$ Here we use the probabilistic expression of NMP to characterize the gas-liquid flow pattern determinism, which expresses as $P_{N M P}=N M P / m !$. $P_{N M P}$ refers to the probability of NMP and $m$ is the embedded dimension.

Figure 5 shows the $P_{N M P}$ under different water velocity when embedded dimensions $m$ are set at 5, 6, and 7 respec- 
tively. We find that the $P_{N M P}$ is related to the value of $m$, but the evolutional characters of $P_{N M P}$ are similar. When gradually increasing the water velocity, the gas-liquid two-phase flow patterns evolve from slug flow to non-uniform bubble flow and then to the uniform bubble flow. Under very low water velocity, the gas phase coalesces into slugs that are intermittently passing by the sensor in the vertical pipe, leading to regular periodic fluctuations. Hence, the slug flow shows a regular feature. As we can see in Fig. 5, the $P_{N M P}$ of the slug flow keeps a relatively high value, which indicates the deterministic character of slug flow. With increasing water velocity, the gas slugs are broken up into large spherically capped bubbles and the flow pattern evolves into the non-uniform bubble flow. In this situation, the presence of large spherically capped bubbles still shows some intermittent character, which is reflected by the pseudo-periodic fluctuation signals shown in Figs. 4(d)-(f). In this regard, the non-uniform bubble flow also shows a deterministic character that is reflected by a high value of $P_{N M P}$. Uniform bubble flow occurs at high water velocity when large spherically capped bubbles are broken into uniformly distributed small gas bubbles. The fluctuations of the uniform bubble flow are more stochastic-like and the motion of the gas bubbles tends to be more random. From Fig. 5, we find that the $P_{N M P}$ of the uniform bubble flow gradually decreases with increasing water velocity. This indicates that the higher water velocity (higher turbulence energy) results in a more uniform and dispersed distribution of the gas bubbles. This also indicates that the determinism of uniform bubble flow is strongly correlated with the flow condition. tion signals when the embeded dimension $m$ is 6 . The average value of $P_{N M P}$ gradually decreases from slug flow to non-uniform bubble flow and then to uniform bubble flow, indicating the gradual reduction of the determinism degree with the flow pattern evolution. The variations of $P_{N M P}$ also reveal the flow pattern determinism dynamics. As shown in Fig. 6, the $P_{N M P}$ variation of the slug flow is smaller than that of other two flow patterns. It means that the determinism of the slug flow is not sensitive to the flow condition, i.e, the fluid velocity. Probably the determinism of the slug flow only depends on the intermittent motion of gas slugs. However, the bubble size and distribution of the uniform bubble flow is strongly depending on the water velocity. Much higher water velocity leads to more dispersed bubble distribution. Hence, the determinism of the uniform bubble flow varies with flow conditions and the range of $P_{N M P}$ is much larger than that of the slug flow. The variation of non-uniform bubble flow seems somewhere in between that of the slug flow and of the uniform bubble flow.

FIG. 6. The box-whisker plot of $P_{N M P}$ under different flow patterns.

\section{B. Scaling behaviors of NMP with the flow pattern evolution}

Scaling behaviors are strongly related to the complex system dynamics. A stochastic system generates quite different scaling dynamics from a deterministic system. Hence, the scaling behaviors of fluid fluctuation signals can be used to characterize the gas-liquid two-phase flow pattern determinism evolutional dynamics. Figure 7 shows the scaling behaviors with respect to different gas-liquid two-phase flow patterns when $m$ is set as 6 . We find that the $P_{N M P}$ decreases

The box-whisker plot of Fig. 6 shows the determinism based on the statistical analysis of the experimental fluctua- 
with the length of the analyzed time series. Furthermore, the decay rate of the $P_{N M P}$ is associated with flow pattern evolution. For fixed gas velocity, the increased water flow velocity means higher turbulence energy and the gas phase tends to be more dispersed and have a stochastic distribution. This is also shown in Fig. 7, the $P_{N M P}$ decreases faster at higher water velocity, which indicates less determinism. The fluid determinism is gradually decreased when the flow pattern evolves from slug to non-uniform bubble flow and then to uniform bubble flow.

FIG. 7. The scaling behavior of $P_{N M P}$ with flow pattern evolution.

The stretched exponential model, ${ }^{48}$ given in (3), is an effective indicator of the NMP scaling behavior. We use two fitting parameters for this model which are the decay rate $B$ and the stretching exponent $\gamma$ to characterize the gas-liquid twophase flow pattern deterministic evolutional dynamics. Figure 8 shows the NMP decay rate and the stretching exponent under different flow conditions. When increasing the water velocity, the value of the decay rate $B$ and the stretching exponent $\gamma$ exhibit opposite trends. We also find that when the flow pattern evolves from non-uniform bubble flow to uniform bubble flow, the stretching exponent $\gamma$ starts to increase while the decay rate $B$ starts to decrease. This indicates that the mixed gas-liquid system is changing from deterministic to stochastic, when the flow pattern transition occurs from non-uniform bubble flow to uniform bubble flow.

To obtain a more detailed understanding of the gas-liquid two-phase flow pattern evolutional dynamics, we construct the joint-distribution of the decay rate $B$ and the stretching exponent $\gamma$. As shown in Fig. 9, most points of the slug flow are located in region 1, where the value of the decay rate $B$ is much higher (the value is around 0.8 ) and the stretching exponent
FIG. 8. The evolution characteristics of the decay rate and the stretching exponent when increasing the water velocity.

$\gamma$ is low (below 0.01), indicating that the slug flow exhibits a deterministic dynamics. When the flow pattern evolves from the slug flow to the non-uniform bubble flow, the mixed fluid still exhibits a regular character due to the intermittent presence of large spherically capped bubbles. However, the determinism of the non-uniform bubble flow begins to slightly decrease, which can be shown by the data distribution of the nonuniform bubble flow. As we can see in region 2, where most data points of the non-uniform bubble flow are located, both the decay rate of NMP and the stretching exponent are slightly decreased as compared to that of the slug flow. As for the region 3 where most data points of the uniform bubble flow are located, both the decay rate of NMP and the stretching exponent reach relatively low value, indicating that the determinism is further decreased when the flow pattern evolves to the uniform bubble flow. Also, we notice that the range of region 3 is wider than that of region 1 and region 2 . This means that the determinism of the uniform bubble flow is quite sensitive to the flow condition, i.e, the fluid velocity. The joint distribution of the decay rate $B$ and the stretching exponent $\gamma$ gives a clear demonstration of the gas-liquid two-phase flow pattern determinism evolutional dynamics. It could potentially be a useful tool to reveal the evolutional dynamics of more complex fluid.

\section{v. CONCLUSION}

In this work, we study the gas-liquid two-phase flow system determinism with indicators that are related to missing ordinal 
flow, are expected based on our work.

\section{ACKNOWLEDGMENTS}

This work was supported by National Natural Science Foundation of China under Grant Nos. 41704131.

\section{DATA AVAILABILITY}

The data that support the findings of this study are available from the corresponding author upon reasonable request.

${ }^{1}$ W. Liu and B. Bai, "Transition from bubble flow to slug flow along the streamwise direction in a gas-liquid swirling flow," Chem. Eng. Sci. 202, 392-402 (2019)

${ }^{2}$ C. Tan, Y. Shen, K. Smith, F. Dong, and J. Escudero, "Gas-liquid flow pattern analysis based on graph connectivity and graph-variate dynamic connectivity of ert," IEEE Trans. Instrum. Meas. 68, 1590-1601 (2018).

${ }^{3}$ I. Zadrazil and C. N. Markides, "An experimental characterization of liquid films in downwards co-current gas-liquid annular flow by particle image and tracking velocimetry," Int. J. Multiphase Flow 67, 42-53 (2014).

${ }^{4}$ X. Zhou, B. Doup, and X. Sun, "Measurements of liquid-phase turbulence in gas-liquid two-phase flows using particle image velocimetry," Meas. Sci. Technol. 24, 125303 (2013).

FIG. 9. The joint distribution of the decay rate of NMP and the stretching exponent under different flow patterns.

patterns. First, a gas-liquid two-phase flow experiment is conducted in a vertical $50 \mathrm{~mm}$ inner diameter pipe. With the aid of high speed camera, we observe three typical gas-liquid twophase flow patterns which are the slug flow, the non-uniform bubble flow and the uniform bubble flow. In addition, the fluid conductance fluctuation signals are collected for our analyses of the flow pattern determinism and the evolutional character.

We characterize the determinism of different gas-liquid flow ordinal patterns with the probability of the number of missing patterns $\left(P_{N M P}\right)$. The results show that $P_{N M P}$ of the slug flow and the non-uniform bubble flow keeps a relatively high value, indicating deterministic flow characteristics of these two flow patterns. $P_{N M P}$ of the uniform bubble flow is smaller than that of the slug flow and the non-uniform bubble flow, showing that the determinism of the uniform bubble flow is reduced. We also find that the determinism of the uniform bubble flow is very sensitive to the flow conditions.

In addition, we investigate the flow pattern evolutional dynamics with the stretched exponential model. The joint distribution of the decay rate $B$ and the stretching exponent $\gamma$ is constructed to characterize the gas-liquid two-phase flow pattern determinism. The results show that the gas-liquid two-phase system determinism is slightly reduced when the flow pattern evolves from the slug flow to the non-uniform bubble flow. However, when the flow pattern changes to the uniform bubble flow, there exists determinism reduction, which probably is attribute to a more dispersed gas bubble distribution. Our research provides a new application of missing ordinal patterns in the field of two-phase flow pattern analysis. Broader applications in more complex fluid system, such as multi-phase tactless bubble/slug velocity measurement method of gas-liquid two-phase flow in small channels," IEEE Trans. Instrum. Meas. 68, 3253-3267 (2018). ${ }^{6} \mathrm{~N}$. Mithran and V. Muniyandi, "Ir transceiver irradiation characteristics on bubble/slug flow regimes in conventional and minichannels," IEEE Trans. Instrum. Meas. 68, 240-249 (2018)

${ }^{7}$ S. Agahzamin and L. Pakzad, "Cfd investigation of the gas dispersion and liquid mixing in bubble columns with dense vertical internals," Chem. Eng. Sci. 203, 425-438 (2019).

${ }^{8}$ Deendarlianto, M. Andrianto, A. Widyaparaga, O. Dinaryanto, Khasani, and Indarto, "Cfd studies on the gas-liquid plug two-phase flow in a horizontal pipe," J. Pet. Sci. Eng. 147, 779 - 787 (2016)

${ }^{9}$ E. Z. Massoud, Q. Xiao, and H. A. El-Gamal, "Numerical study of an individual taylor bubble drifting through stagnant liquid in an inclined pipe," Ocean Eng. 195, 106648 (2020)

${ }^{10} \mathrm{X}$. Lu, P. Xie, D. B. Ingham, L. Ma, and M. Pourkashanian, "A porous media model for cfd simulations of gas-liquid two-phase flow in rotating packed beds," Chem. Eng. Sci. 189, 123 - 134 (2018).

${ }^{11}$ W. L. Li, Y. Ouyang, X. Y. Gao, C. Y. Wang, S. Lei, and Y. Xiang, "Cfd analysis of gas-liquid flow characteristics in a microporous tube-in-tube microchannel reactor," Comput. Fluids 170, 13-23 (2018).

${ }^{12}$ R. Kiran, R. Ahmed, and S. Salehi, "Experiments and cfd modelling for two phase flow in a vertical annulus," Chem. Eng. Res. Des. 153, 201-211 (2020).

${ }^{13}$ M. Parsi, M. Agrawal, V. Srinivasan, R. E. Vieira, C. F. Torres, B. S. McLaury, S. A. Shirazi, E. Schleicher, and U. Hampel, "Assessment of a hybrid cfd model for simulation of complex vertical upward gas-liquid churn flow," Chem. Eng. Res. Des. 105, 71-84 (2016).

${ }^{14}$ F. Franca, M. Acikgoz, R. T. Lahey Jr, and A. Clausse, "The use of fractal techniques for flow regime identification," Int. J. Multiphase Flow 17, 545 -552 (1991).

${ }^{15}$ B. Wu, A. S. Ribeiro, M. Firouzi, T. E. Rufford, and B. Towler, "Use of pressure signal analysis to characterise counter-current two-phase flow regimes in annuli," Chem. Eng. Res. Des. 153, 547-561 (2020).

${ }^{16}$ V. S. Chalgeri and J. H. Jeong, "Flow regime identification and classification based on void fraction and differential pressure of vertical twophase flow in rectangular channel," Int. J. Heat Mass Transfer 132, 802-816 (2019).

${ }^{17}$ Z. Gao, W. Dang, C. Mu, Y. Yang, S. Li, and C. Grebogi, "A novel multiplex network-based sensor information fusion model and its application to industrial multiphase flow system," IEEE Trans. Ind. Inform. 14, 39823988 (2017). 
${ }^{18} \mathrm{O}$. C. Jones Jr and N. Zuber, "The interrelation between void fraction fluctuations and flow patterns in two-phase flow," Int. J. Multiphase Flow 2, 273-306 (1975).

${ }^{19} \mathrm{M}$. A. Vince and R. T. Lahey Jr, "On the development of an objective flow regime indicator," Int. J. Multiphase Flow 8, 93 - 124 (1982).

${ }^{20}$ Z. Gao, Y. Yang, L. Zhai, N. Jin, and G. Chen, "A four-sector conductance method for measuring and characterizing low-velocity oil-water two-phase flows," IEEE Trans. Instrum. Meas. 65, 1690-1697 (2016).

${ }^{21}$ V. T. Nguyen, D. J. Euh, and C. H. Song, "An application of the wavelet analysis technique for the objective discrimination of two-phase flow patterns," Int. J. Multiphase Flow 36, 755 - 768 (2010).

${ }^{22}$ W. Zhang, C. Wang, and H. Wang, "Hilbert-huang transform-based electrostatic signal analysis of ring-shape electrodes with different widths," IEEE Trans. Instrum. Meas. 61, 1209-1217 (2011).

${ }^{23}$ Q. Y. Yang, Y. F. Han, W. X. Liu, H. X. Zhang, Y. Y. Ren, and N. D. Jin, "Investigation on the effect of polymer in vertical oil-water two-phase flow using nonlinear analysis," Exp. Therm. Fluid Sci. 80, 1 - 18 (2017).

${ }^{24}$ Z. K. Gao, S. S. Zhang, W. D. Dang, S. Li, and Q. Cai, "Multilayer network from multivariate time series for characterizing nonlinear flow behavior," Int. J. Bifurcation Chaos 27, 1750059 (2017).

${ }^{25} \mathrm{~T}$. Wei, X. Li, and D. Wang, "Identification of gas-liquid two-phase flow patterns in dust scrubber based on wavelet energy entropy and recurrence analysis characteristics," Chem. Eng. Sci. 217, 115504 (2020).

${ }^{26}$ Z. Gao, H. Wang, W. Dang, Y. Li, X. Hong, M. Liu, and G. Chen, "Complex network analysis of wire-mesh sensor measurements for characterizing vertical gas-liquid two-phase flows," IEEE Trans. Circuits Syst. II-Express Briefs (2019).

${ }^{27}$ Z. K. Gao, W. D. Dang, L. Xue, and S. S. Zhang, "Directed weighted network structure analysis of complex impedance measurements for characterizing oil-in-water bubbly flow," Chaos 27, 035805-035805 (2017).

${ }^{28}$ W. Dang, Z. Gao, L. Hou, D. Lv, S. Qiu, and G. Chen, "A novel deep learning framework for industrial multiphase flow characterization," IEEE Trans. Ind. Inform. 15, 5954-5962 (2019).

${ }^{29}$ C. Cozin, F. E. C. Vicencio, F. A. de Almeida Barbuto, R. E. M. Morales, M. J. da Silva, and L. V. R. Arruda, "Two-phase slug flow characterization using artificial neural networks," IEEE Trans. Instrum. Meas. 65, 494-501 (2016).

${ }^{30}$ L. Wang, J. Liu, Y. Yan, X. Wang, and T. Wang, "Gas-liquid two-phase flow measurement using coriolis flowmeters incorporating artificial neural network, support vector machine, and genetic programming algorithms," IEEE Trans. Instrum. Meas. 66, 852-868 (2016).

${ }^{31}$ H. Ji, J. Long, Y. Fu, Z. Huang, B. Wang, and H. Li, "Flow pattern identification based on emd and ls-svm for gas-liquid two-phase flow in a minichannel,' IEEE Trans. Instrum. Meas. 60, 1917-1924 (2011).

${ }^{32}$ M. Du, N. D. Jin, Z. K. Gao, and B. Sun, "Analysis of total energy and time-frequency entropy of gas-liquid two-phase flow pattern," Chem. Eng. Sci. 82, 144-158 (2012)

${ }^{33}$ Y. F. Han, N. D. Jin, L. S. Zhai, Y. Y. Ren, and Y. S. He, "An investigation of oil-water two-phase flow instability using multivariate multi-scale weighted permutation entropy," Phys. A 518, 131-144 (2019).

${ }^{34}$ M. Du, X. Y. Chen, H. Y. Liu, Q. He, R. X. Bai, W. X. Liu, and Z. W.

$\mathrm{Li}$, "Time irreversibility from time series for analyzing oil-in-water flow transition," Math. Probl. Eng. 2016 (2016)

${ }^{35}$ J. M. Amigo, S. Zambrano, and M. A. F. Sanjuan, "Detecting determinism in time series with ordinal patterns: A comparative study," Int. J. Bifurcation Chaos Appl. Sci. Eng. 20, 2915-2924 (2010).

${ }^{36}$ L. C. Carpi, P. M. Saco, and O. A. Rosso, "Missing ordinal patterns in correlated noises," Phys. A 389, 2020 - 2029 (2010).

${ }^{37}$ J. B. Borges, H. S. Ramos, R. A. F. Mini, O. A. Rosso, A. C. Frery, and A. A. F. Loureiro, "Learning and distinguishing time series dynamics via or- dinal patterns transition graphs," Appl. Math. Comput. 362, 124554 (2019).

${ }^{38} \mathrm{C}$. W. Kulp and L. Zunino, "Discriminating chaotic and stochastic dynamics through the permutation spectrum test," Chaos 24, 033116 (2014).

${ }^{39}$ A. Ji and P. Shang, "Analysis of financial time series through forbidden patterns," Phys. A 534, 122038 (2019).

${ }^{40}$ L. Rong and P. Shang, "Evaluation of missing ordinal pattern and its fractional distribution entropy," Phys. A 537, 122317 (2020).

${ }^{41}$ G. Ouyang, C. Dang, D. A. Richards, and X. Li, "Ordinal pattern based similarity analysis for eeg recordings," Clin. Neurophysiol. 121, 694-703 (2010).

${ }^{42}$ I. Echegoyen, V. Vera-Ávila, R. Sevilla-Escoboza, J. H. Martínez, and J. M. Buldú, "Ordinal synchronization: Using ordinal patterns to capture interdependencies between time series," Chaos, Solitons Fractals 119, 8 18 (2019).

${ }^{43}$ Y. Yin, X. Wang, Q. Li, P. Shang, and F. Hou, "Quantifying interdependence using the missing joint ordinal patterns," Chaos 29, 073114 (2019).

${ }^{44}$ S. Fischer, A. Schumann, and A. Schnurr, "Ordinal pattern dependence between hydrological time series," J. Hydrol. 548, 536-551 (2017).

${ }^{45}$ M. Xu, P. Shang, and A. Lin, "Multiscale recurrence quantification analysis of order recurrence plots," Phys. A 469, 381-389 (2017).

${ }^{46}$ A. A. B. Pessa and H. V. Ribeiro, "Characterizing stochastic time series with ordinal networks,” Phys. Rev. E 100 (2019).

${ }^{47}$ F. Olivares, L. Zunino, and O. A. Rosso, "Quantifying long-range correlations with a multiscale ordinal pattern approach,” Phys. A 445, 283-294 (2016).

${ }^{48}$ F. Olivares, L. Zunino, M. C. Soriano, and D. G. Pérez, "Unraveling the decay of the number of unobserved ordinal patterns in noisy chaotic dynamics," Phys. Rev. E 100, 042215 (2019).

${ }^{49}$ C. Grebogi and A. N. Kaufman, "Decay of statistical dependence in chaotic orbits of deterministic mappings," Phys. Rev. A 24, 2829 (1981).

${ }^{50} \mathrm{C}$. Bandt and B. Pompe, "Permutation entropy: a natural complexity measure for time series," Phys. Rev. Lett. 88, 174102 (2002).

${ }^{51}$ F. Traversaro, F. O. Redelico, M. R. Risk, A. C. Frery, and O. A. Rosso, "Bandt-pompe symbolization dynamics for time series with tied values: A data-driven approach," Chaos 28, 075502 (2018).

${ }^{52}$ N. Rubido, C. Grebogi, and M. S. Baptista, "Entropy-based generating markov partitions for complex systems," Chaos 28, 033611 (2018).

${ }^{53}$ F. Takens, "Detecting strange attractors in turbulence," in Dynamical systems and turbulence, Warwick 1980 (Springer, 1981) pp. 366-381.

${ }^{54}$ R. Bowen, "Markov partitions for axiom a diffeomorphisms," Am. J. Math 92, 725-747 (1970).

${ }^{55}$ C. W. Kulp, J. M. Chobot, B. J. Niskala, and C. J. Needhammer, "Using forbidden ordinal patterns to detect determinism in irregularly sampled time series," Chaos 26, 023107-023107 (2016).

${ }^{56}$ C. W. Kulp, L. Zunino, T. Osborne, and B. Zawadzki, "Using missing ordinal patterns to detect nonlinearity in time series data," Phys. Rev. E 96, 022218 (2017).

${ }^{57}$ F. Olivares, L. Zunino, and D. G. Pérez, "Revisiting the decay of missing ordinal patterns in long-term correlated time series," Phys. A 534, 122100 (2019).

${ }^{58}$ M. Du, N. D. Jin, Z. K. Gao, Z. Y. Wang, and L. S. Zhai, "Flow pattern and water holdup measurements of vertical upward oil-water two-phase flow in small diameter pipes," Int. J. Multiphase Flow 41, 91-105 (2012).

${ }^{59}$ C. S. Daw, C. E. A. Finney, M. Vasudevan, N. A. van Goor, K. Nguyen, D. D. Bruns, E. J. Kostelich, C. Grebogi, E. Ott, and J. A. Yorke, "Selforganization and chaos in a fluidized bed," Phys. Rev. Lett. 75, 2308 (1995). 


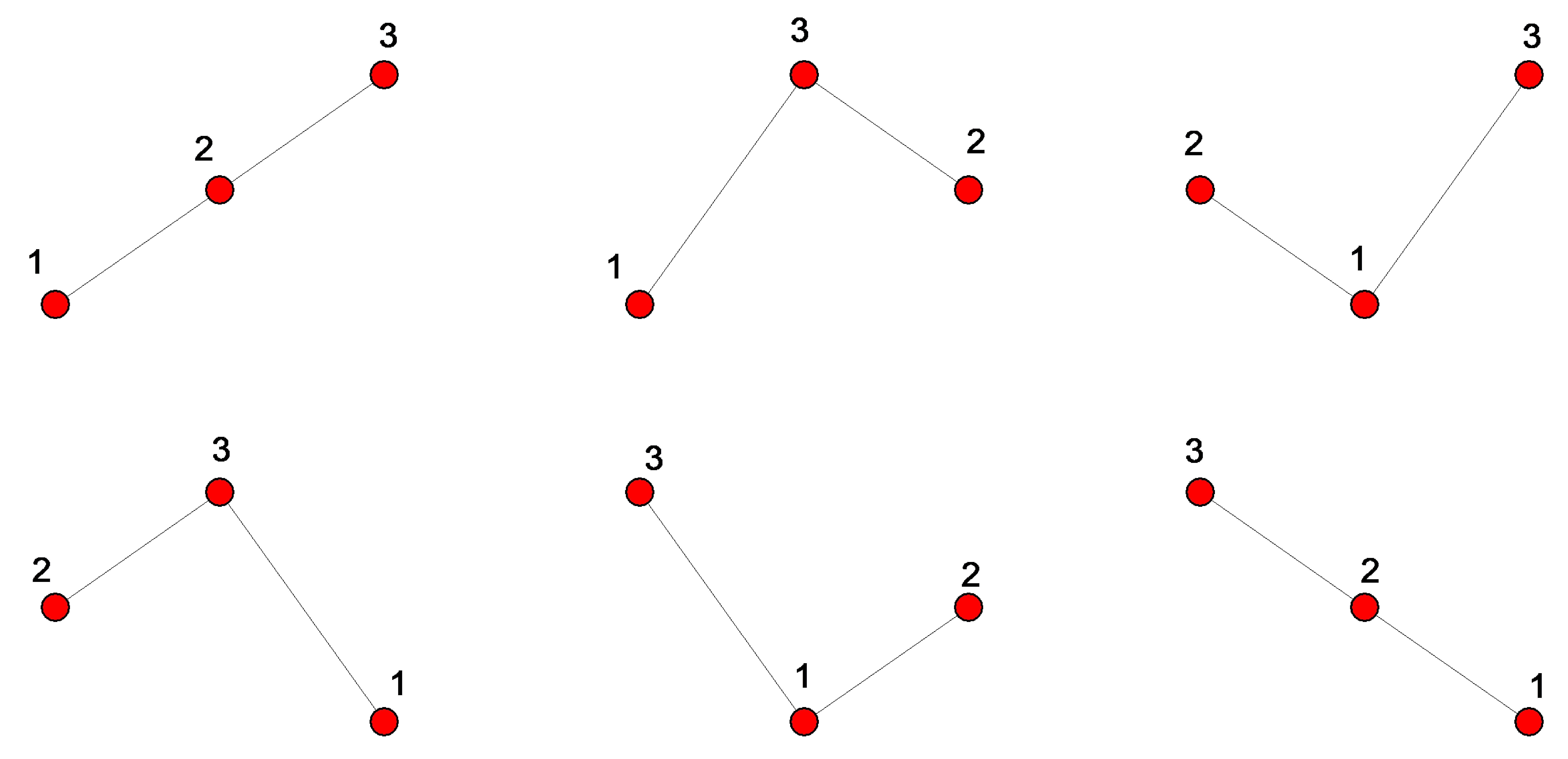




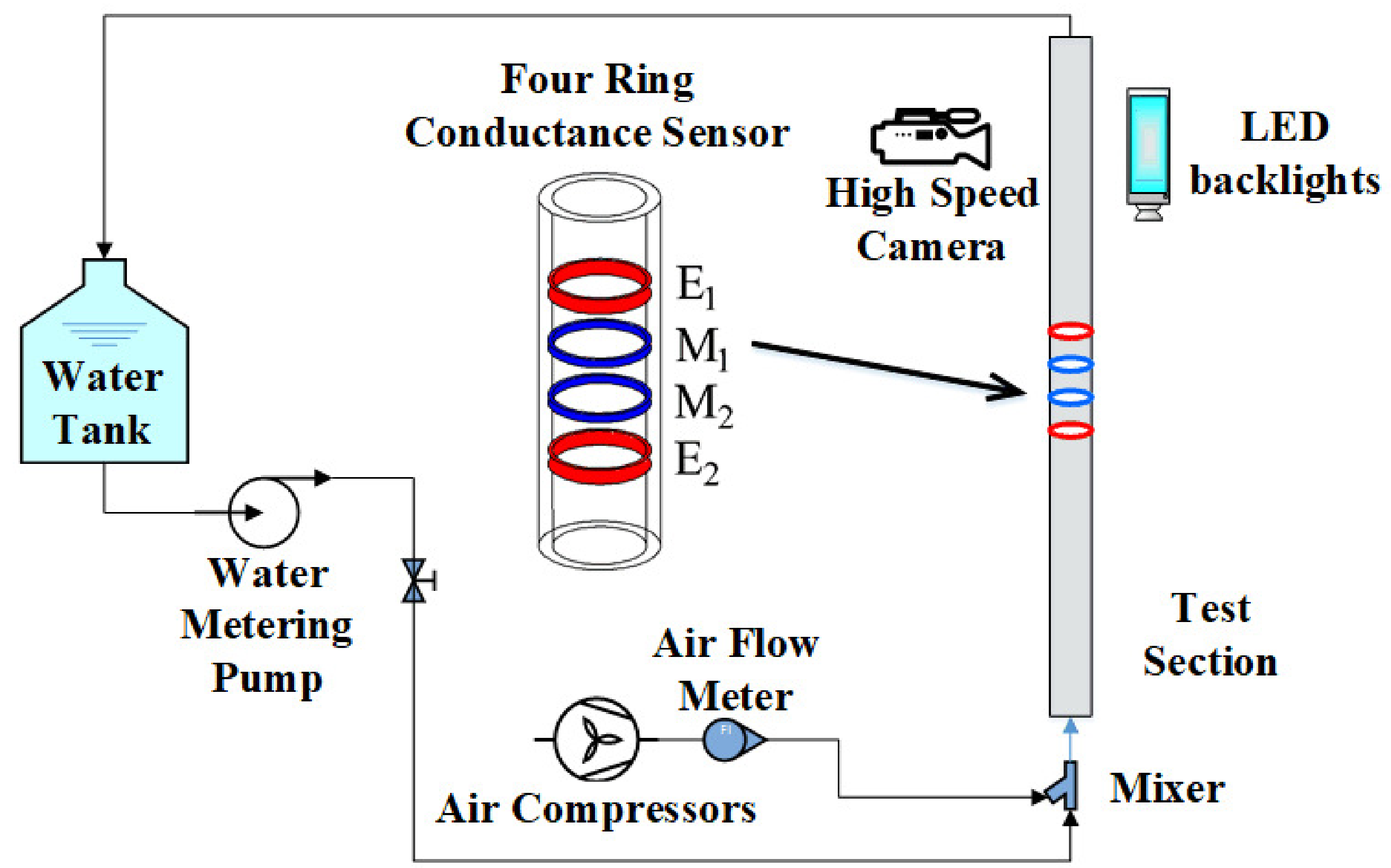


Flow pattern evolve with time

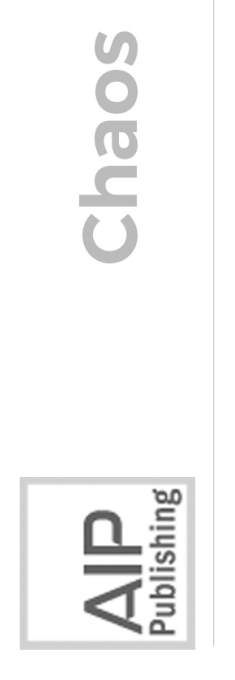

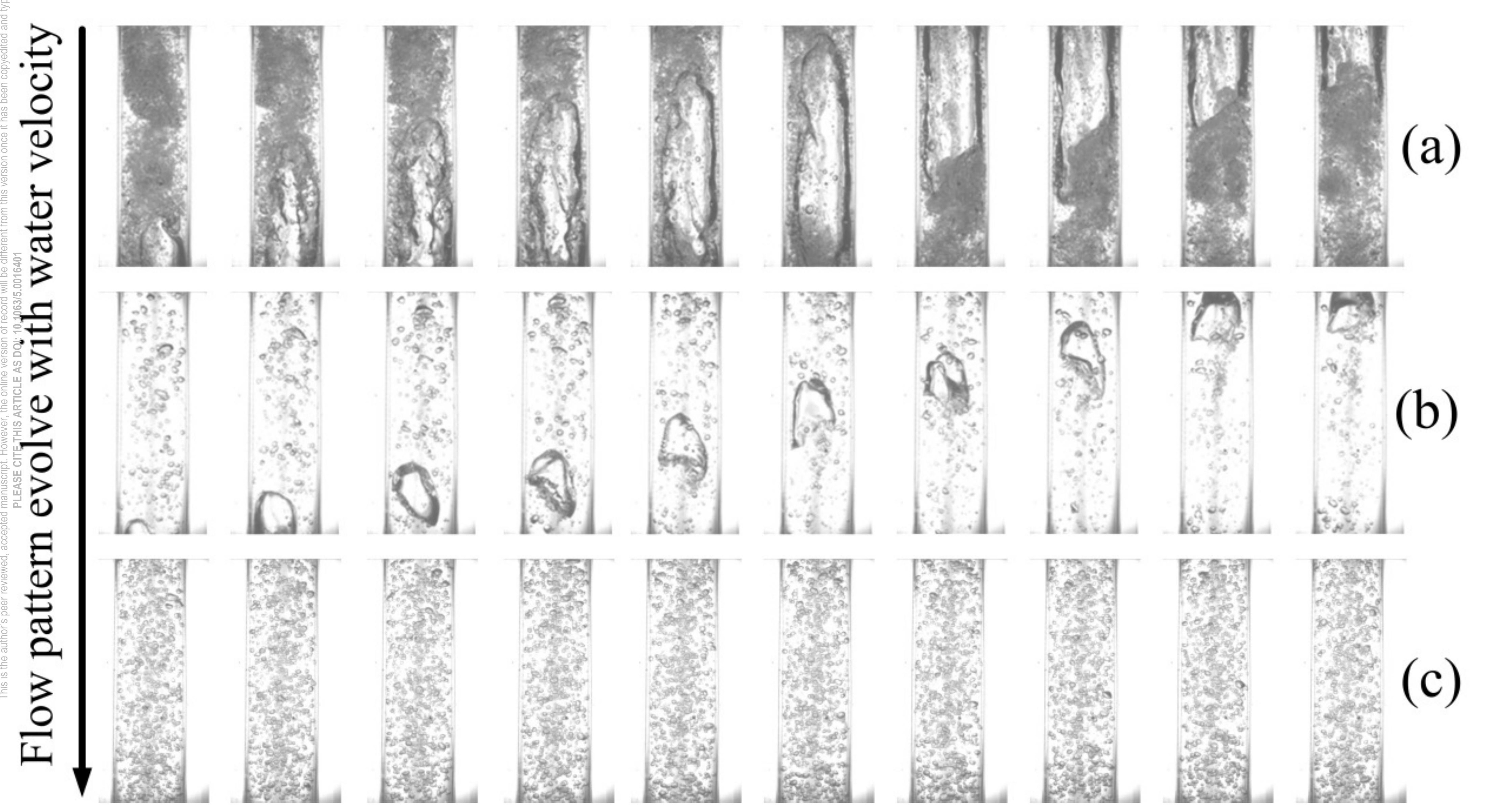


$U g:$ Gas velocity $\quad U w$ : Water velocity

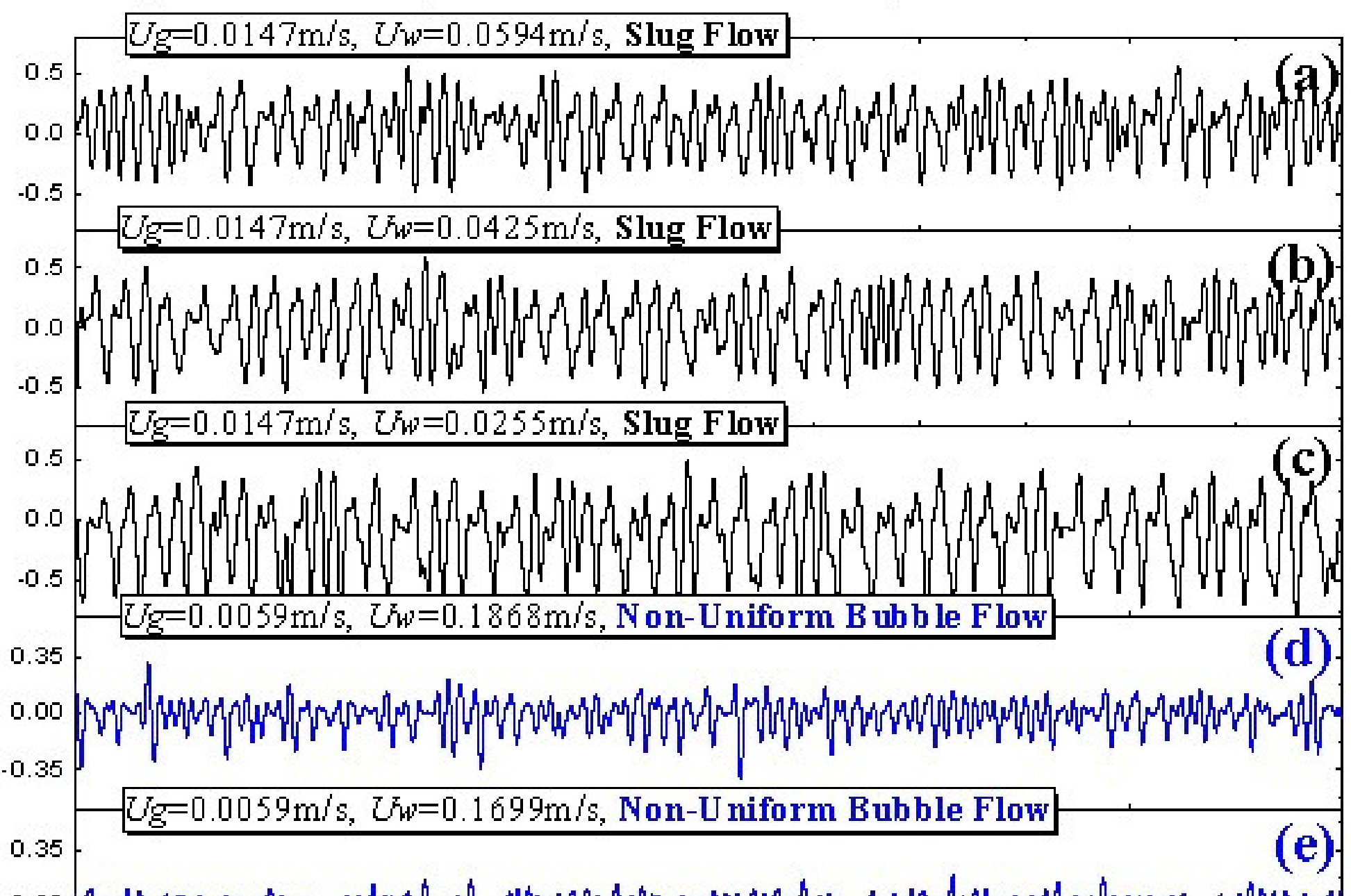

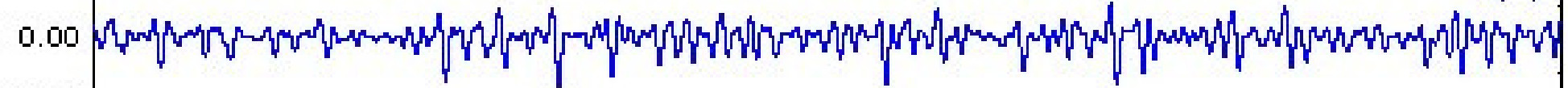
.0 .35

0.35 Ug=0.0059m/s, Uw=0.1529m/s, Non-Uniform B ubble Flow

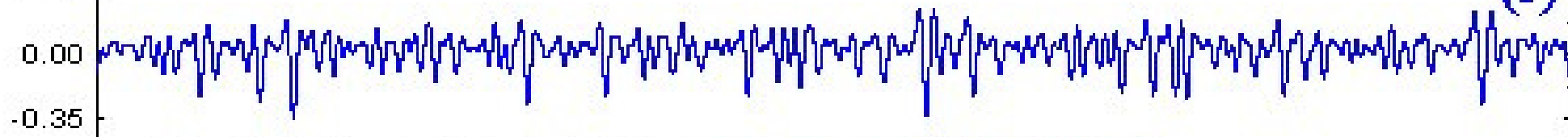

0.035

$U g=0.0041 \mathrm{~m} / \mathrm{s}, U_{w}=0.1699 \mathrm{~m} / \mathrm{s}$, Uniform Bubble Flow

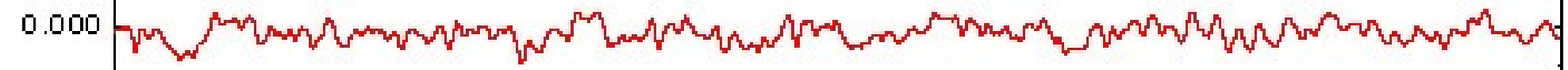

$-0.035$

0.035

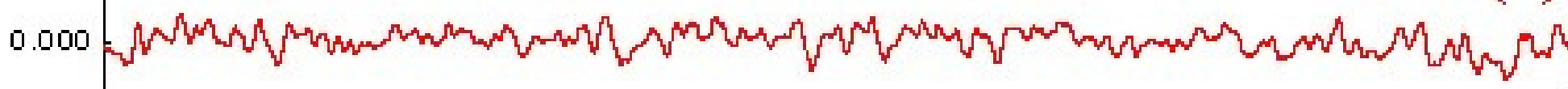

$-0.035$

0.035

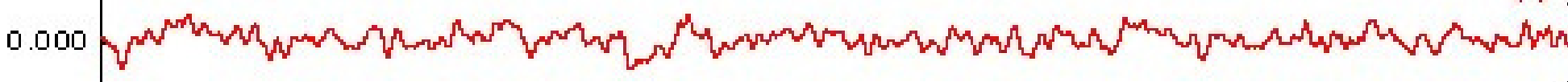
$-0.035$

\begin{tabular}{lllllll}
\hline 0 & 5 & 10 & 15 & 20 & 25 & 30
\end{tabular}

Time(s) 




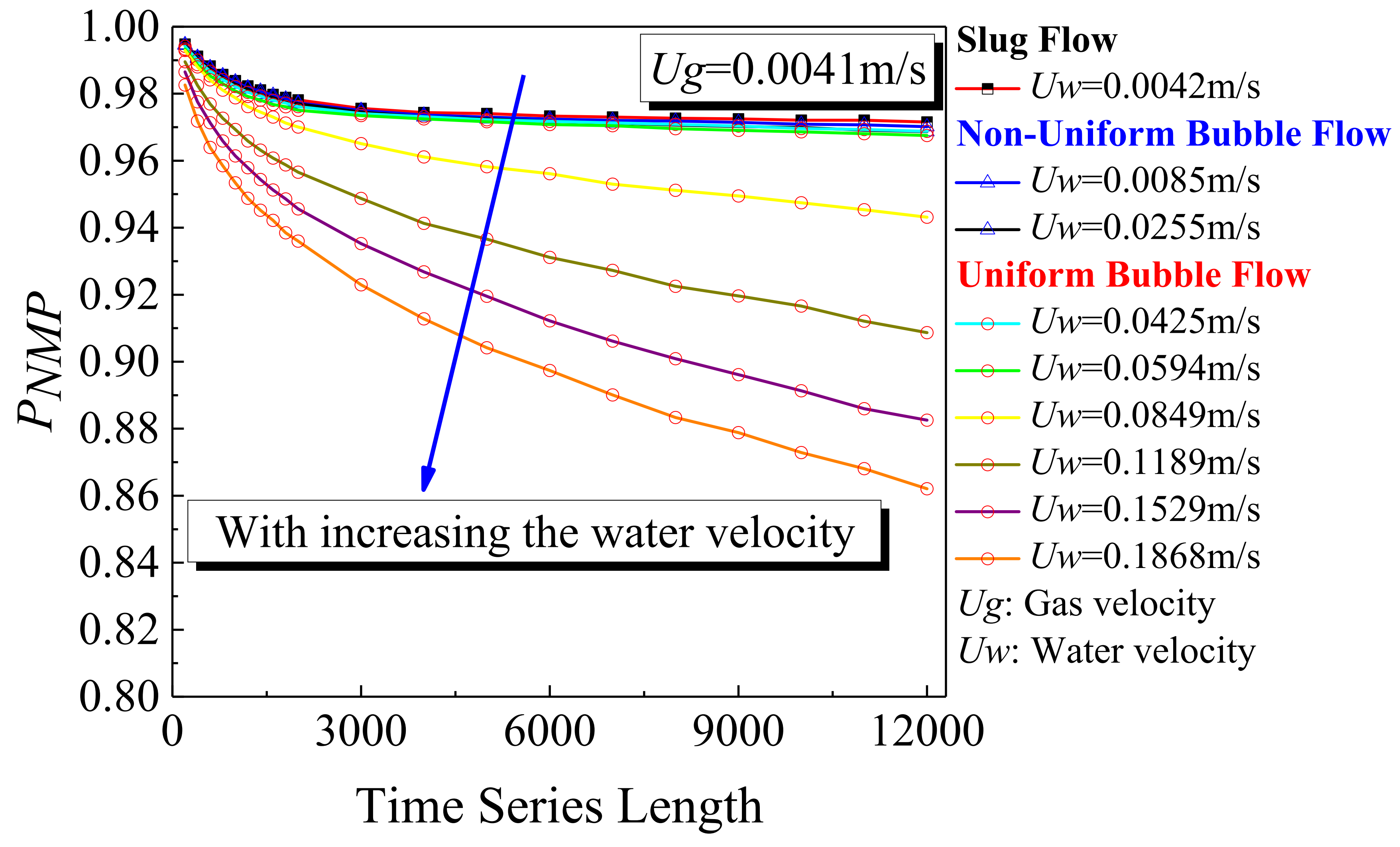



